Disclosure of Interest: None declared DOI: 10.1136/annrheumdis-2018-eular.7186

\section{AB0034 LOKING FOR A SLE SIGNATURE ON PERIPHERAL B CELL SUBSETS: DOES A PREPONDERANT CD38 +PLASMABLAST-SUBPOPULATION LACK CD73 AS A SIGN OF A DISTURBED ADENOSINE AXIS?}

M. Siekierka-Harreis, M. Schroedter, G. Chehab, J. Richter, M. Schneider, G. Pongratz. Rheumatology, Heinrich-Heine University Duesseldorf, Medical Faculty, Duesseldorf, Germany

Background: Systemic Lupus Erythematosus (SLE) is an autoimmune disorder characterised by polyclonal Bcell activation, production of dsDNA-autoantibodies and cytokines. Subsets of Bcells play a central role in SLE-pathogenesis. The inflammatory milieu is characterised by the accumulation of adenosine, which confers immunosuppressive effects.

Objectives: In SLE, the role of CD73, an enzyme involved in the extracellular generation of adenosine from ATP, is not well characterised. This study aimed to characterise expression of CD73 Bcell subsets of SLE-patients as compared to healthy controls $(\mathrm{HC})$.

Methods: Bcell subsets were characterised from peripheral blood of 23 SLE patients attending the outpatient clinic at the Rheumatology Unit of University Hospital Düsseldorf and of $15 \mathrm{HC}$ by FACS. All patients fulfilled the revised SLEcriteria of ACR and were randomly collected in clinical remission state (SLEDAI $1.1 \pm 1.9)$.

Results: By comparison of Bcell subsets between SLE and HC, CD38 was dominantly expressed by SLE-patients (SLE $74.2 \% \pm 12.9 \%$ vs. HC $64.2 \%$ $\pm 12.2 \%$; $p(M W U)=0.018)$. Furthermore, SLE-patients showed an increase in CD19 + lgD CD27+CD38 high plasmablasts (SLE 2.1\% $\$ 3.4 \%$ vs $\mathrm{HC} 0,4 \%$ $\pm 0.4 \%, \mathrm{p}(\mathrm{MWU})<0.001)$. Furthermore, SLE-plasmablasts showed decreased CD73 expression as compared to HC(SLE $2.1 \% \pm 1.9 \%$ vs HC $3.5 \% \pm 2.2 \%$; p $(\mathrm{MWU})=0.034)$. SLE-Bcells revealed a trend towards an augmented CD38highCD138+plasmacell fraction (SLE $0.40 \% \pm 0.5 \%$ vs HC $0.08 \%$ $\pm 0.07 \%$; $p=0.07$ ), without any difference in CD73 expression. On the other hand, exhausted-memory B cell fraction (CD19 + IgD CD27-CD21-CD138-), showed an increased CD73 expression in SLE (SLE 13.7\% $\pm 9.2 \%$ vs HC $6.2 \%$ $\pm 5.4 \% ; p=0.004)$.

Conclusions: Our study confirms CD38 +plasmablasts as being increased in peripheral blood from SLE patients as compared to HC. Furthermore, the data reveal a deficiency for CD73 on SLE plasmablasts, which suggests a decreased anti-inflammatory capacity of SLE plasmablasts as compared to $\mathrm{HC}$, supporting the notion of a disturbed adenosine axis in SLE. On the other hand, the enlarged CD73 +exhausted memory pool in SLE could point to an accelerated flow of CD73 +B cells into an exhausted B cell fraction. These findings support the hypothesis of dysregulation of the adenosine axis in SLE even in inactive SLE patients.

\section{REFERENCES:}

[1] Szabo K, Papp G, Szanto A, Tarr T, Zeher M. A comprehensive investigation on the distribution of circulating follicular $T$ helper cells and $B$ cell subsets in primary Sjogren's syndrome and systemic lupus erythematosus. Clinical and experimental immunology 2016;183(1):76-89.

[2] Jacobi AM, Reiter K, Mackay M, Aranow C, Hiepe F, Radbruch A, et al. Activated memory $B$ cell subsets correlate with disease activity in systemic lupus erythematosus: delineation by expression of CD27, IgD, and CD95. Arthritis and Rheumatism 2008:58(6):1762-73.

[3] Deaglio S, Dwyer KM, Gao W, Friedman D, Usheva A, Erat A, et al. Adenosine generation catalyzed by CD39 and CD73 expressed on regulatory $T$ cells mediates immune suppression. The Journal of Experimenta Medicine 2007;204(6):1257-65.

[4] Hasko G, Linden J, Cronstein B, Pacher P. Adenosine receptors: therapeutic aspects for inflammatory and immune diseases. Nature Reviews Drug Discovery 2008;7(9):759-70.

Disclosure of Interest: None declared DOI: 10.1136/annrheumdis-2018-eular.4451
$\mathrm{AB} 0035$

A PHYSIOLOGICAL NETWORK OF IGG AUTOANTIBODIES TARGETING G PROTEIN COUPLED RECEPTORS

O.C. Marques $^{1}$, A. Marques ${ }^{1}$, R. de Vito ${ }^{2}$, L.M. Giil ${ }^{3}$, J. Rademacher ${ }^{4,5}$, J. Günther ${ }^{4,5}$, G. Marschner ${ }^{1}$, S. Pitann ${ }^{1}$, S. Adler ${ }^{6}$, D. Dragun ${ }^{7}$, P. Lamprecht ${ }^{1}$ A. Müller ${ }^{1}$, X. Yu ${ }^{8}$, F. Petersen ${ }^{8}$, H. Heidecke ${ }^{9}$, B.E. Engelhardt ${ }^{2}$, G. Riemekasten ${ }^{1}$ ${ }^{1}$ Reumathology, University of Lübeck, 23562 Lübeck, Germany; ${ }^{2}$ Computer Science, University of Princeton, Princeton, USA; ${ }^{3}$ Deaconess Hospital, University of Bergen, Bergen, Norway, ${ }^{4}$ Reumathology, Charité University Hospital; ${ }^{5}$ Cell Autoimmunity Group, German Rheumatism Research Center, Berlin, Germany; ${ }^{6}$ Reumathology, University Hospital Bern, Bern, Switzerland; ${ }^{7}$ Dept. of Nephrology and Cardiovascular Research, Campus Virchow, Charité University Hospital, Berlin; ${ }^{8}$ Research Center Borstel, Airway Research Center North (ARCN), Members of the German Center for Lung Research (DZL), Borstel; ${ }^{9}$ CellTrend $\mathrm{GmbH}$, Luckenwalde, Germany

Background: Since the time when Paul Ehrlich conceived the term "horror autotoxicus", autoantibodies have been associated with the development of autoimmune diseases. However, several works have recently shown the presence of autoantibodies in sera from healthy subjects $(n=489)$, who do not develop autoimmune diseases.

Objectives: Here, we report a network of immunoglobulin $\mathrm{G}(\mathrm{lgG})$ autoantibodies targeting G protein-coupled receptors (GPCRs), growth factors and growth factorrelated molecules in sera from healthy subjects.

Methods: Autoantibody levels in sera were assessed using ELISA. Autoantibody network was analysed by exploratory factor analysis (EFA), dandelion plo method, hierarchical clustering, and multi-study factor analysis (MFA). We also reverse engineered autoantibody functions through in silico evaluation of autoantibody target interactions using STRING and gene ontology (GO). To test the autoantibodies functionality we assessed the in vitro production of IL- 8 by PBMCs and neutrophil migration in response to $\mathrm{IgG}$ from healthy subjects as well as ETAR immunised and control mice. Leukocyte cellularity to secondary immune organs was analysed comparing ETAR-immunised with control mice.

Results: Gender, age and the presence of pathological conditions (systemic sclerosis $n=84$, Alzheimer's disease $n=91$ and ovarian cancer $n=207$ ) changed corre lations between the autoantibodies and their hierarchical clustering distribution. Notably, subjects at age below and above 65 years or with pathological conditions exhibited particular autoantibody hierarchical clustering signatures. In addition, females at age above 65 years, representing the group of subjects with higher risk to develop SSc, displayed the closest link to SSc in terms of autoantibody hierarchical clustering. Finally, autoantibody directed against the endothelin receptor type A (ETAR) showed an essential role in the autoantibody network by orches trating neutrophil trafficking in vitro and in ETAR-immunised mice.

Conclusions: Our data provide a framework for the existence of a physiological network of autoantibodies and reveal a new paradigmatic view on these physiological molecules.

Disclosure of Interest: None declared

DOI: 10.1136/annrheumdis-2018-eular.4461

\section{$\mathrm{AB} 0036$ \\ ROLE OF PROGRAMMED DEATH-1 PATHWAY ON CD8+ T CELLS CYTOTOXICITY IN PRIMARY BILIARY CHOLANGITIS}

S. Zhang, X. Tao, L. Wang, L. Zhao, J. Sun, Z. Chen, H. Chen, F. Zhang. Peking Union Medical College Hospital, Beijing, China

Background: Primary Biliary Cholangitis (PBC) is a chronic progressive autoim mune disease. It has been proven that there was abnormal activation of CD $8+T$ cells. Previous studies have shown that abnormal expression programmed death 1 (PD-1) and its ligand (PD-L1) in PBC. However, no study was found to confirm the abnormality of PD-1/PD-L1 pathway in CTL in PBC.

Objectives: To investigate the role of PD-1 and its ligand PD-L1 on CD8 ${ }^{+} T$ cells cytotoxicity in the immunological mechanism of PBC

Methods: The expression of PD- 1 in peripheral CD8 ${ }^{+} \mathrm{T}$ cells of 69 patients diag nosed with $\mathrm{PBC}$ as well as 58 health controls $(\mathrm{HC})$ was detected by flow cytometry. Plasma cytokines related to PD-1/PD-L1 pathway were detected by ELISA. The co-localization of PD-1 and CD-8, PD-L1 and CK19 in portal region in liver biopsy was analysed by immunofluorescence assay. Meanwhile, the relative gene expression of PD-1 regulatory pathway in $\mathrm{CD}^{+} \mathrm{T}$ lymphocytes were meas ured by RT-PCR. And co-culture of PD- $1^{ \pm} \mathrm{CD} 8^{+} \mathrm{CTL}$ and HiBEC was done in vitro to detect the cytotoxicity, proliferation and cytokine expression of $C D 8^{+} \mathrm{CTL}$ and the apoptosis of HiBEC.

Results: The proportion of peripheral PD- $1^{+} \mathrm{CD} 8^{+} \mathrm{T}$ cell decreased in PBC $(12.0 \% \pm 8.8 \%)$ than $\mathrm{HC}(19.9 \% \pm 12.5 \%)(\mathrm{p}<0.001)$. The plasma concentration of $\mathrm{IL}-10, \mathrm{IFN}-\gamma$ and TGF- $\beta$ in the PBC group were higher than that in $\mathrm{HC}(8.29$ $\pm 9.00 \mathrm{pg} / \mathrm{ml}$ v.s. $4.43 \pm 5.08 \mathrm{pg} / \mathrm{ml}, \mathrm{p}=0.0066 ; 51.94 \pm 52.94$ vs $26.71 \pm 26.26 \mathrm{pg} / \mathrm{ml}$, $\mathrm{p}=0.0015 ; 1302.0 \pm 1972.8 \mathrm{pg} / \mathrm{ml}$ vs $205.8 \pm 298.9 \mathrm{pg} / \mathrm{ml}, \mathrm{p}=0.0018$, resp.). Compared with $\mathrm{HC}(\mathrm{n}=19)$, Tbet gene expression in $\mathrm{CD}^{+}$T lymphocyteswas increase 
in PBC group $(n=21)(0.82 \pm 0.76$ vs $3.03 \pm 4.23, p=0.028)$. Immunofluorescence co-localization revealed that increased PD-1 positive cells in early PBC stage than late one, and less PD-L1 positive cells as well as PD-L1 and CK19 co-localised ones in PBC patients compared to HC. In the CTL and HiBEC co-culture system in vitro, the cytotoxicity of $\mathrm{PD}-1^{+} \mathrm{CD} 8^{+} \mathrm{T}$ cells was weaker, with less proliferation and tendency of decreased production of IFN- $\gamma$ and TGF- $\beta$ compared to PD-1 $1^{-} \mathrm{CD} 8^{+} \mathrm{T}$ cells. Meawhile, HiBEC apoptosis was relatively more in PD-1 $\mathrm{CD} 8^{+} \mathrm{T}$ cells co-cluture group. These effects could be antagonised by anti-PD-1 antibody and enhanced by PD-L1.

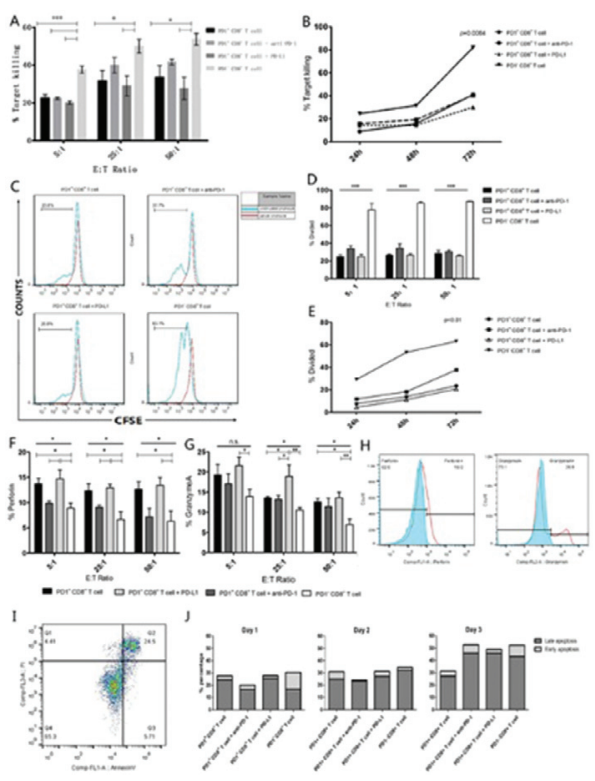

Abstract AB0036 - Figure 1

Conclusions: In PBC, the expression of Tbet is up-regulated in $\mathrm{CD}^{+} \mathrm{T}$ cells, which leads to the down-regulated expression of PD-1 on. Meanwhile, the expression of PD-L1 in HiBEC may be down-regulated. The silenced PD-1/PD-L1 pathway caused more $C D 8^{+} T$ cells proliferation, more related cytokines production and the enhanced CTL cytotoxic effects on HiBEC. PD-1/PD-L1 pathway functions as an important pathway in the immunological mechanism of PBC.

\section{REFERENCES:}

[1] Kaplan MM. N Engl J Med, 1996:335(21):1570-1580.

[2] [2] Kita H, et al. J Clin Invest, 2002;109(9):1231-1240.

[3] [3] Yang GX, et al. J Immunol, 2011;186(2):1259-1267.

[4] [4] Keir ME, et al. Annu Rev Immunol, 2008;26:677-704.

[5] [5] Mataki N, et al. Am J Gastroenterol, 2007;102(2):302-312.

Acknowledgements: This work was supported in part by a grant from Capital Youth Medical Research and Development Fund (No. 2016-4-40111), National Natural Science Foundation (Outstanding Youth Foundation) (No. 81501414) and a grant from The National Key Research and Development Program of China ${ }^{\text {No. }}$ ${ }^{2016}$ YFA010100)

Disclosure of Interest: None declared

DOI: 10.1136/annrheumdis-2018-eular.1859

\section{AB0037 MIR-155 PROMOTES THE DIFFERENTIATION OF TH17 CELL BY TARGETING ETS-1 IN RHEUMATOID ARTHRITIS}

Z. Yin ${ }^{1}$, W. He ${ }^{1}$, X. Chen ${ }^{1}$, X. Luo ${ }^{1}$, Y. Zhang ${ }^{1,2}$, Z. Ye ${ }^{1} .{ }^{1}$ Shenzhen Futian Hospital for Rheumatic Diseases, Shenzhen; ${ }^{2}$ Rheumatology and Immunology, First Clinical College of Harbin Medical University, Harbin, China

Background: MicroRNAs play important roles in arthritis. MiR-155 emerges as a central regulator of the immune response, but its function in autoimmune arthritis is poorly understood.

Objectives: To clarify the role of miR-155 in the balance of CD4 ${ }^{+} T h 17$ and Treg cell differentiations as well as how their mechanisms function in autoimmune arthritis.

Methods: $\mathrm{CD} 4+\mathrm{T}$ cells were in vitro isolated from DBA1 mice. The transfections of miR-155 mimics and miR-155 inhibitors in CD4 +T cells were performed for enhanced or knockdowns of miR-155 expression and RNA interference was applied to silence Ets-1 gene expression. In addition, we carried out in vivo experiments of enhanced miR-155 expression by lenti-virus mediated miR-155 (LVmiR-155) infection and silencing of miR-155 expression by lenti-virus-anti-miR155(LV-anti-miR-155) within our well-established type II collagen (CII)-induced arthritis (CIA) mice model. The ratios of $\mathrm{IL}-17^{+} \mathrm{TH} 17$ cells were analysed using flow cyctometry (FCM). The expressions of some key autoimmune-response of genes including RORgt, IFN-g, Ets-1, IL-17, IL-23, and Stat3 were further determined by (Taqman) Quantitative Real-time PCR and Western blotting. Initially $\mathrm{n}=6$ per group if not indicated further:totally 6 groups in parallel per experiment. ${ }^{*} p<0.05$, considered as significantly; ${ }^{* *} p<0.01$, considered as highly significantly. Results: For the first time we showed that miR-155 may promote Th17 cell differentiation in RA pathogenesis. Firstly, we observed that miR-155 may significantly induce the expressions of some key autoimmune-response genes including RORgt, IFN-g, IL-17, IL-23, and Stat3 but it significantly inhibited Ets-1 gene expression. 2ndly, miR-155 may in part modulates Th17 cell differentiation by targeting Ets-1 in that indeed the expression of miR-155 significantly co-related with disease index in CIA mice. Furthermore, the CIA mice with in vivo forced expression of miR-155 have significantly more Th17 cells(i.e.high ratio) and severe CIA disease index compared to their control CIA mice;strikingly, they also have a significantly higher expression of aforementioned autoimmune-response genes compared to their corresponding controls along with the response of Ets-1 exactly in a verse direction. Consistently, the CIA mice with in vivo knockdown of miR-155 expression resulted in significantly less Th17 cells and lesser severe CIA disease index compared to their control CIA mice. However, they have a significantly lower expression of above-mentioned autoimmune-response genes compared to their corresponding controls along with a response of Ets-1 in a verse direction (the data in part shown in Figure 1 ;sham $=\mathrm{ClA}$ treated with LV-nonspecific sequence). Conclusions: For the first time our data show miR-155 has a critical role in Th17 differentiation and the RA pathogenesis via targeting the Ets-1. Although it warrants further investigations, miR-155 might be a promising therapeutic target for autoimmune diseases, esp. RA.

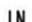

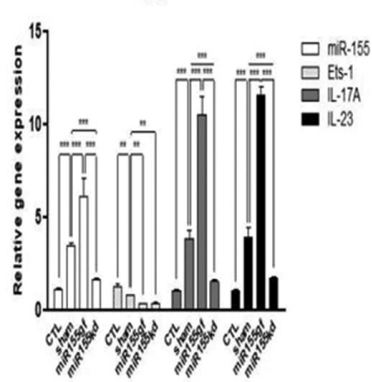

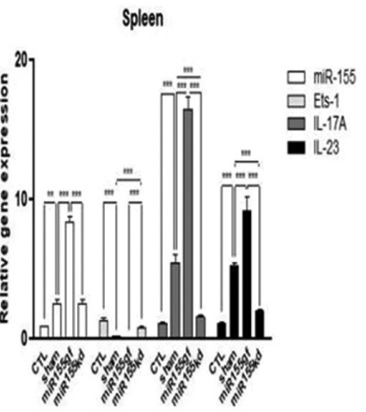

Abstract AB0037 - Figure 1
Acknowledgements: This work ws supported by National Natural Science Foundation of China \#81102266. All authors listed were either involved in conception and design, or acquisition, analysis and interpretation of data, and/or writing. Each author gave their final approval of this version.

Disclosure of Interest: None declared

DOI: 10.1136/annrheumdis-2018-eular.3877

\section{Innate immunity in rheumatic diseases}

\section{AB0038 TBK1: A KEY REGULATOR AND POTENTIAL TREATMENT TARGET FOR INTERFERON POSITIVE SYSTEMIC LUPUS ERYTHEMATOSUS AND SYSTEMIC SCLEROSIS}

E. Huijser ${ }^{1}$, I. Bodewes ${ }^{1}$, C. van Helden-Meeuwsen ${ }^{1}$, L. Tas ${ }^{1}$, R. Huizinga ${ }^{1}$, V. Dalm², M. van Hagen ${ }^{2}$, N. Groot ${ }^{3}$, S. Kamphuis ${ }^{3}$, P. van Daele ${ }^{2}$, M. Versnel' ${ }^{1}$ Immunology, ${ }^{2}$ Internal Medicine; ${ }^{3}$ Pediatric Rheumatology, Erasmus MC, Rotterdam, Netherlands

Background: Upregulation of type I interferons (IFN-I) is a hallmark of systemic autoimmune diseases like systemic lupus erythematosus (SLE) and systemic sclerosis (SSc). Three different receptor families are implicated in the induction of IFN-I production: Toll-like receptors (TLRs), RIG-like receptors (RLRs) and DNAsensing receptors (DSRs). TANK-binding kinase (TBK1), is an important signalling hub downstream of RLRs and DSRs. TBK1 activates IRF3 and IRF7, leading 